\title{
Effectivity and Power
}

Citation for published version (APA):

Karos, D., \& Peters, H. (2016). Effectivity and Power. Maastricht University, Graduate School of Business and Economics. GSBE Research Memoranda No. 034 https://doi.org/10.26481/umagsb.2016034

\section{Document status and date:}

Published: 01/01/2016

DOI:

10.26481/umagsb.2016034

Document Version:

Publisher's PDF, also known as Version of record

\section{Please check the document version of this publication:}

- A submitted manuscript is the version of the article upon submission and before peer-review. There can be important differences between the submitted version and the official published version of record.

People interested in the research are advised to contact the author for the final version of the publication, or visit the DOI to the publisher's website.

- The final author version and the galley proof are versions of the publication after peer review.

- The final published version features the final layout of the paper including the volume, issue and page numbers.

Link to publication

\footnotetext{
General rights rights.

- You may freely distribute the URL identifying the publication in the public portal. please follow below link for the End User Agreement:

www.umlib.nl/taverne-license

Take down policy

If you believe that this document breaches copyright please contact us at:

repository@maastrichtuniversity.nl

providing details and we will investigate your claim.
}

Copyright and moral rights for the publications made accessible in the public portal are retained by the authors and/or other copyright owners and it is a condition of accessing publications that users recognise and abide by the legal requirements associated with these

- Users may download and print one copy of any publication from the public portal for the purpose of private study or research.

- You may not further distribute the material or use it for any profit-making activity or commercial gain

If the publication is distributed under the terms of Article $25 \mathrm{fa}$ of the Dutch Copyright Act, indicated by the "Taverne" license above, 


\section{Maastricht University}

Dominik Karos, Hans Peters

\section{Effectivity and Power}

RM/16/034

\section{GSBE}

Maastricht University School of Business and Economics

Graduate School of Business and Economics

P.O Box 616

NL-6200 MD Maastricht

The Netherlands 


\title{
Effectivity and Power
}

\author{
Dominik Karos* Hans Peters ${ }^{\dagger}$
}

August 30, 2016

\begin{abstract}
We axiomatically develop a class of power indices for effectivity functions, both for the case where the set of alternatives is finite and where it is infinite. Such power indices make it possible to take the issues under consideration into account, in contrast to power indices defined just for simple games. As an example, we consider the US legislative system. We also show that our approach can be used to develop power indices for spatial political games.
\end{abstract}

Keywords: Effectivity Function, Power Index

JEL classification: C71

\section{Introduction}

A power index is a tool to measure and compare the power of players in political and economic situations. In a democratic Parliament the number of seats of a political party is a poor indication of its power. Rather, a measure of power should be based on the likelihood that such a party is decisive. One way to assess this is to model Parliament as a simple game, by establishing which coalitions of political parties are winning and which are losing - based on the required majorities to pass bills or amendments. To such a simple game one may then apply a power index, for instance the Banzhaf-Coleman index or Shapley-Shubik index (see for instance Bertini et al., 2013, for a recent overview).

Similarly, economic situations as for instance financial and corporate governance structures may be modelled by simple games, and power indices may be applied in order to obtain some indication of the real influence of firms or investment companies in such situations (Crama and Leruth, 2007, 2013; Karos and Peters, 2015).

This approach to power measurement clearly has its limitations. In political situations, neither the position of a political party nor the issues at stake are

\footnotetext{
* Department of Economics, University of Oxford, United Kingdom. Email: dominik. karos@seh.ox.ac.uk.

${ }^{\dagger}$ Department of Quantitative Economics, Maastricht University, The Netherlands. Email: h.peters@maastrichtuniversity.nl.
} 
taken into consideration. For this reason, in political science spatial models are considered, which enable to represent both a party's position and the issues involved.

In the present paper we generalize the simple game approach and also the spatial game approach by considering the very broad model of an effectivity function (Moulin and Peleg, 1982) and developing a theory of power indices for such functions. Given a number of players and a set of alternatives, an effectivity function assigns to each coalition (subset of players) a collection of sets of alternatives. If a coalition $S$ is effective for a set $B$ of alternatives, then this is generally interpreted as the coalition $S$ being able to guarantee that the final alternative is in $B$. Alternatives could be social states, and a coalition may be constitutionally entitled to the final social state being in a certain set of social states. Or alternatives could be laws, and a coalition of parties may have the power (e.g., because it has a certain majority) to pass certain laws. In financial or governance structures, coalitions of shareholders may enforce certain decisions within companies if they own sufficiently many shares. More formally, effectivity functions may be derived from social choice functions or correspondences, or from game forms.

The following is a specific political example, which will recur at several places in this paper.

Example 1.1 (The US Legislative Process). In US legislation any bill must be passed both by the Senate (100 members) and the House (of Representatives, 435 members) and signed by the President before it comes to law. If the President vetoes the bill, the Congress can override this decision by a two third majority. In case of an Executive Order by the President, no approval by the Congress is needed.

The President can agree on international treaties, but these must be approved by a two third majority of the Senate. In case of Congressional-executive Agreements (agreements that affect domestic policies, such as free trade agreements) a simple majority approval both in the Senate and in the House are necessary; and Executive Agreements need no approval by the Congress at all.

Currently, the Republicans have a majority in both chambers of the Congress, but they do not have a two third majority. If we consider the Republicans $(R)$ and the Democrats $(D)$ as one player each, we see that the singleton set $\{D\}$ is effective for any outcome that can be reached via Executive Orders or Agreements, while $\{R\}$ is not effective for anything.

Alternatively, we can analyze the situation from the individuals' points view. In this case the singleton $\{p\}$ which contains only the president, is effective for any outcome that can be reached via Executive Orders or Agreements; a coalition $S$ is effective for an outcome $\{a\}$ that can be reached via a domestic law or a Congressional-executive Agreement if $S$ contains $p$ and more than half of the members of the Senate and of the House. In the first case it is also sufficient if $S$ contains more than two thirds of the members of the Senate and of the House. In particular, a coalition $T$ is effective for the set $A \backslash\{a\}$ - in other words, $T$ can veto $a$ - if $p \in T$ and, in case of a domestic law, $T$ contains more than one third 
of the members of either the Senate or the House. If $a$ is an international treaty then $S$ is effective for $\{a\}$ if $p \in S$ and $S$ contains two thirds of the members of the Senate.

Effectivity functions and the concept of a power index for effectivity functions are introduced in Section 2. Our basic axioms for power indices are introduced in Section 3: the Transfer Property, Anonymity, and (Strong) Monotonicity. The Transfer Property is a generalized additivity condition which enables us to unravel an effectivity function in terms of elementary effectivity functions which are much easier to analyze. Anonymity and Monotonicity are natural conditions in this context. In Section 4 we consider the case where the set of alternatives is finite and characterize all power indices satisfying the Transfer Property, Anonymity, and Monotonicity. Such a power index works as follows. For each set of alternatives, consider the simple game in which exactly those coalitions that are effective for that set of alternatives are winning. Also, to each set of alternatives attach a non-negative number such that these numbers sum up to one. Then the power index is obtained by taking the sum of the Shapley values of these simple games weighted by the chosen numbers. This leaves much freedom, namely in choosing these weights. In the remainder of Section 4 we (1) show that all these power indices reduce to the Shapley value for an effectivity function associated with a simple game (winning coalitions are effective for every set of alternatives, losing coalitions only for the whole set); (2) establish connections between power indices for the duals of simple games and polar effectivity functions (which describe what coalitions can obtain in reaction to outside players); (3) show that a power index is neutral if and only if the weights only depend on the cardinalities of the sets of alternatives; (4) show that requiring Strong Monotonicity results in non-singleton nontrivial sets of alternatives having weight zero; and (5) consider the role of null-players. In Section 5 we extend our main characterization to effectivity functions for an infinite set of alternatives, under Strong Monotonicity instead of Monotonicity and, additionally a Null Player axiom and a continuity condition. Basically, we obtain a similar characterization, now with the weights of the finite case replaced by a probability measure over the set of alternatives. In a separate subsection, we show how this approach can lead to developing power indices for spatial political models, similar in spirit to the Owen-Shapley spatial power index.

In an Appendix we show that the axioms in our main characterization are logically independent.

\section{Preliminaries}

We start with some notations. For a set $D$ we denote by $P(D)$ the set of all subsets of $D$, and by $P_{0}(D)$ the set of all nonempty subsets of $D$. By $|D|$ we denote the number of elements of $D$.

Throughout, $N=\{1, \ldots, n\}(n \in \mathbb{N})$ is the set of players. Subsets of $N$ are also called coalitions. We denote by $A$ the set of alternatives. We fix a set 
$\mathcal{T} \subseteq P_{0}(A)$. In the first part of the paper, $A$ is finite and $\mathcal{T}=P_{0}(A)$; later, $A$ can be infinite, endowed with a topology, and then $\mathcal{T}$ will be the collection of nonempty closed subsets of $A$.

Definition 2.1. An effectivity function (for $\mathcal{T}$ ) is a map $E: P(N) \rightarrow P(\mathcal{T})$ such that (i) $P(\emptyset)=\emptyset$, (ii) $A \in E(S)$ for every $S \in P_{0}(N)$, and (iii) $E(N)=\mathcal{T}$. An effectivity function $E$ is monotonic if $B \in E(S)$ implies $B^{\prime} \in E(T)$ for all $B, B^{\prime} \in \mathcal{T}$ and $S, T \in P_{0}(N)$ such that $B \subseteq B^{\prime}$ and $S \subseteq T$. An effectivity function $E$ is superadditive if $B \cap B^{\prime} \in E(S \cup T)$ for all $B, B^{\prime} \in \mathcal{T}$ and $S, T \in$ $P_{0}(N)$ such that $B \in E(S), B^{\prime} \in E(T)$, and $S \cap T=\emptyset$. By $\mathcal{E}$ we denote the set of all monotonic and superadditive effectivity functions.

For $E, F \in \mathcal{E}$ define the operations $\cap$ and $\cup$ by

$$
\begin{aligned}
& (E \cup F)(S)=E(S) \cup F(S) \\
& (E \cap F)(S)=E(S) \cap F(S)
\end{aligned}
$$

for all $S \in P(N)$. The following result is well-known, and we omit its easy proof.

Lemma 2.2. Let $E, F \in \mathcal{E}$. Then $E \cap F \in \mathcal{E}$.

Note that $E, F \in \mathcal{E}$ does not imply $E \cup F \in \mathcal{E}$ since $E \cup F$ is monotonic but not necessarily superadditive.

The aim of this paper is to develop a class of power indices for effectivity functions.

Definition 2.3. A power index is a $\operatorname{map} \varphi: \mathcal{E} \rightarrow \mathbb{R}^{N}$ with $\sum_{i \in N} \varphi_{i}(E)=1$.

With effectivity functions, simple games can be associated. A simple game is a function $v: 2^{N} \rightarrow\{0,1\}$ satisfying $v(\emptyset)=0, v(N)=1$, and $v(S) \leq v(T)$ for all $S, T \in P(N)$ with $S \subseteq T$ - the last property is called monotonicity. Coalitions $S$ with $v(S)=1$ are winning, the other coalitions are losing. The Shapley value $S h$, also called Shapley-Shubik power index (Shapley, 1953; Shapley and Shubik, 1954), assigns to player $i$ in a simple game $v$ the number

$$
S h_{i}(v)=\sum_{S \in P(N): i \notin S} \frac{|S| !(n-|S|-1) !}{n !}[v(S \cup\{i\})-v(S)] .
$$

For an effectivity function $E \in \mathcal{E}$, a set of alternatives $B \in \mathcal{T}$, and a coalition $S \in P(N)$ let

$$
v_{B}^{E}(S)= \begin{cases}1 & \text { if } B \in E(S) \\ 0 & \text { otherwise }\end{cases}
$$

Clearly, $v_{B}^{E}$ is a simple game for each $B \in \mathcal{T}$. In this game, a coalition is winning exactly if it is effective for $B$. 
For two simple games $v, w$ we define the simple games $v \vee w$ and $v \wedge w$ by

$$
\begin{aligned}
& (v \vee w)(S)=\max (v(S), w(S)), \text { and } \\
& (v \wedge w)(S)=\min (v(S), w(S)) .
\end{aligned}
$$

The following lemma, the proof of which is left to the reader, will be useful later on.

Lemma 2.4. Let $E, F$ be two effectivity functions. Then for all $B \in \mathcal{T}$ :

$$
\begin{aligned}
& \left(v_{B}^{E} \vee v_{B}^{F}\right)=v_{B}^{E \cup F}, \text { and } \\
& \left(v_{B}^{E} \wedge v_{B}^{F}\right)=v_{B}^{E \cap F} .
\end{aligned}
$$

Example 2.5. Recall the US Legislation example from the beginning. Let $N=\{p\} \cup S \cup H$ where $p$ is the president, $S$ contains the 100 members of Senate, and $H$ contains the 435 members of the House. Let $A_{1}$ be the set of outcomes that can be achieved by international treaties, $A_{2}$ those that can be achieved by Congressional-executive Agreements, $A_{3}$ those that can be achieved by Executive Agreements or Orders, and $A_{4}$ those that can be achieved by domestic laws. Then Legislation can be represented by the minimal effectivity function $E$ with

$$
\begin{array}{ll}
\{a\} \in E(T) \text { for all } a \in A_{3} & \text { if } p \in T, \\
\{a\} \in E(T) \text { for all } a \in A_{4} & \text { if }|T \cap S| \geq 67 \text { and }|T \cap H| \geq 290, \\
\{a\} \in E(T) \text { for all } a \in A_{1} \cup A_{3} & \text { if } p \in T \text { and }|T \cap S| \geq 67, \\
\{a\} \in E(T) \text { for all } a \in A_{2} \cup A_{3} \cup A_{4} & \text { if } p \in T \text { and }|T \cap S| \geq 51 \\
& \quad \text { and }|T \cap H| \geq 218, \\
\{a\} \in E(T) \text { for all } a \in A & \text { if } p \in T \text { and }|T \cap S| \geq 67 \\
& \text { and }|T \cap H| \geq 218, \\
A \backslash\{a\} \in E(T) \text { for all } a \in A_{1} & \text { if }|T \cap S| \geq 34, \\
A \backslash\{a\} \in E(T) \text { for all } a \in A_{2} \cup A_{4} & \text { if }|T \cap S| \geq 50 \text { or }|T \cap H| \geq 218, \\
A \backslash\{a\} \in E(T) \text { for all } a \in A & \text { if } p \in T \text { and }(|T \cap S| \geq 34 \\
& \quad \text { or } T \cap H \geq 146) .
\end{array}
$$

Note that this effectivity function is somewhat simplified as it ignores the majority leaders' ability to schedule the consideration of a bill. The combination of a Filibuster by a Senator (which can be broken by a majority of 60 Senators) together with a Pocket Veto by the President is covered by the last line above.

\section{Main axioms for power indices}

The main condition that we will put on a power index $\varphi$ is the following, which was first formulated for a value on simple games by Dubey (1975).

Transfer Property For all $E, F \in \mathcal{E}$ such that $E \cup F \in \mathcal{E}$,

$$
\varphi(E \cup F)+\varphi(E \cap F)=\varphi(E)+\varphi(F) .
$$


Throughout, we will also impose anonymity. For a permutation $\pi$ of $N$ and an effectivity function $E \in \mathcal{E}$, let $\pi E \in \mathcal{E}$ be defined by $(\pi E)(\pi(S))=E(S)$ for all $S \in P(N)$.

Anonymity $\varphi_{i}(E)=\varphi_{\pi(i)}(\pi E)$ for every $E \in \mathcal{E}$, every permutation $\pi$ of $N$, and every $i \in N$.

Next, we introduce two monotonicity conditions.

Monotonicity $\varphi_{i}(E) \leq \varphi_{i}(F)$ for all $E, F \in \mathcal{E}$ and every $i \in N$ such that $E(S) \backslash E(S \backslash\{i\}) \subseteq F(S) \backslash F(S \backslash\{i\})$ for all $S \in P(N)$.

Strong Monotonicity $\varphi_{i}(E) \leq \varphi_{i}(F)$ for all $E, F \in \mathcal{E}$ and every $i \in N$ such that $\{a\} \in E(S) \backslash E(S \backslash\{i\})$ implies $\{a\} \in F(S) \backslash F(S \backslash\{i\})$ for all $S \in P(N)$ and all $a \in A$.

Monotonicity states that if in one effectivity function a player contributes more than in another one, then in the former that player should be attributed more power than in the latter. Strong monotonicity weakens the premise of monotonicity by requiring a player only to contribute more as far as single alternatives are concerned, and thus results in a stronger axiom. An effectivity function may, for instance, represent the ability of coalitions to keep the social state within a certain range. However, when measuring power it might be more relevant to ask which (single) social states a coalition can achieve, than to which social states a coalition can reduce the outcome. For instance, a party's power in a parliament may be reflected by its ability to pass specific bills. In the effectivity function in Example 2.5 this would mean that the last three cases do not add to the power of any Congressmen. This idea is reflected by strong monotonicity instead of just monotonicity.

Call a player $i \in N$ a null player in $E \in \mathcal{E}$ if $E(S) \backslash E(S \backslash\{i\})=\emptyset$ for all $S \in P(N)$. Note that in particular $E(N \backslash\{i\})=\mathcal{T}$ if $i$ is a null player in $E$. The final condition we introduce in this section is as follows.

Null Player $\varphi_{i}(E)=0$ for every $E \in \mathcal{E}$ and every null player $i$ in $E$.

\section{Finitely many alternatives}

Throughout this section we assume that $A$ is finite and that $\mathcal{T}=P_{0}(A)$. Our main result is the characterization of the set of all power indices satisfying the Transfer Property, Anonymity, and Monotonicity. We start with some preliminary results.

For $B \in P(A)$ we denote $B^{+}=\left\{B^{\prime} \in P_{0}(A): B \subseteq B^{\prime}\right\}$. For an effectivity function $E \in \mathcal{E}$ and a set $B \in P_{0}(A)$ we define

$$
E^{B}(S)= \begin{cases}P_{0}(A) & \text { if } S=N \\ B^{+} & \text {if } B \in E(S) \\ \{A\} & \text { if } B \notin E(S) \text { and } S \neq \emptyset \\ \emptyset & \text { if } S=\emptyset .\end{cases}
$$


Clearly, $E^{B} \in \mathcal{E}$ for each $B \in P_{0}(A)$. We also have the following result about unions of effectivity functions $E^{B}$.

Lemma 4.1. Let $E \in \mathcal{E}$ and let $\emptyset \neq K \subseteq P_{0}(A)$ such that $B \cap B^{\prime} \in K$ for all $B, B^{\prime} \in K$. Then $F=\bigcup_{B \in K} E^{B} \in \mathcal{E}$.

Proof. Clearly, $F$ is monotonic. For superadditivity, let $S, T \in P_{0}(N)$ with $S \cap T=\emptyset, X \in F(S), Y \in F(T)$. Then there are $B, B^{\prime} \in K$ such that $B \subseteq X, B^{\prime} \subseteq Y, B \in E(S)$, and $B^{\prime} \in E(T)$. By superadditivity of $E$ we have $B \cap B^{\prime} \in E(S \cup T)$, and $B \cap B^{\prime} \in K$ since $K$ is closed under intersection. Hence, $X \cap Y \in F(S \cup T)$.

An elementary effectivity function is an effectivity function of the form

$$
U_{T}^{B}(S)= \begin{cases}P_{0}(A) & \text { if } S=N \\ B^{+} & \text {if } S \supseteq T, S \neq \emptyset, \text { and } S \neq N \\ \{A\} & \text { if } S \nsupseteq T \text { and } S \neq \emptyset \\ \emptyset & \text { if } S=\emptyset\end{cases}
$$

for some $B \in P(A)$ and $T \in P(N)$. Clearly, $U_{T}^{B} \in \mathcal{E}$ for all $B \in P(A)$ and $T \in P(N)$. We now show that under the Transfer Property a power index $\varphi$ is completely determined by its values on elementary effectivity functions.

Lemma 4.2. Let $\varphi$ and $\psi$ be power indices satisfying the Transfer Property and let $\varphi\left(U_{S}^{B}\right)=\psi\left(U_{S}^{B}\right)$ for all $B \in P(A)$ and all $S \in P(N)$. Then $\varphi=\psi$.

Proof. Note that any $E \in \mathcal{E}$ can be written as $E=\cup_{B \in P_{0}(A)} E^{B}$. Hence, to prove that $\varphi=\psi$, it is sufficient to prove that $\varphi\left(\cup_{B \in K} E^{B}\right)=\psi\left(\cup_{B \in K} E^{B}\right)$ for every $\emptyset \neq K \subseteq P_{0}(A)$ which is closed under intersection. We prove this by induction on $k=|K|$. First let $k=1$, say $K=\{B\}$, and write $E=E^{B}=$ $\cup_{j \in I} U_{S_{j}}^{B}$, where $I \subseteq \mathbb{N}$ with $|I| \leq 2^{n}$ and $\left\{S_{j} \in P_{0}(N): j \in I\right\}$ is the set of those coalitions $S_{j}$ that are minimally effective for $B$, i.e., $B \in E\left(S_{j}\right)$ but $B \notin E(T)$ for all $T \subsetneq S_{j}$. Then

$$
\begin{aligned}
\varphi(E) & =\sum_{\emptyset \neq J \subseteq I}(-1)^{|J|+1} \varphi\left(\bigcap_{j \in J} U_{S_{j}}^{B}\right)=\sum_{\emptyset \neq J \subseteq I}(-1)^{|J|+1} \varphi\left(U_{\cup_{j \in J}^{B} S_{j}}\right) \\
& =\sum_{\emptyset \neq J \subseteq I}(-1)^{|J|+1} \psi\left(U_{\bigcup_{j \in J}^{B} S_{j}}\right)=\sum_{\emptyset \neq J \subseteq I}(-1)^{|J|+1} \psi\left(\bigcap_{j \in J} U_{S_{j}}^{B}\right) \\
& =\psi(E)
\end{aligned}
$$

where the first and fifth equalities follow from Lemma A.1 in the Appendix. Now let $\hat{k} \geq 2$ and suppose that $\varphi\left(\bigcup_{B \in K} E^{B}\right)=\psi\left(\bigcup_{B \in K} E^{B}\right)$ for every $K$ with $|K|=k<\hat{k}$. Take $\widehat{K}$ with $|\widehat{K}|=\hat{k}$ and let $\widehat{B} \in \widehat{K}$ be a set of maximal 
cardinality. Let $F=\bigcup_{B \in \widehat{K}} E^{B}$. Then by the Transfer Property and Lemmas 2.2 and 4.1 we have

$$
\begin{aligned}
\varphi(F) & =\varphi\left(\bigcup_{B \in \widehat{K} \backslash\{\widehat{B}\}} E^{B}\right)+\varphi\left(E^{\widehat{B}}\right)-\varphi\left(\left(\bigcup_{B \in \widehat{K} \backslash\{\widehat{B}\}} E^{B}\right) \cap E^{\widehat{B}}\right) \\
& =\varphi\left(\bigcup_{B \in \widehat{K} \backslash\{\widehat{B}\}} E^{B}\right)+\varphi\left(E^{\widehat{B}}\right)-\varphi\left(\bigcup_{B \in \widehat{K} \backslash\{\widehat{B}\}} E^{B \cup \widehat{B}}\right) .
\end{aligned}
$$

Thus, by the induction hypothesis,

$$
\varphi(F)=\psi\left(\bigcup_{B \in \widehat{K} \backslash\{\widehat{B}\}} E^{B}\right)+\psi\left(E^{\widehat{B}}\right)-\psi\left(\bigcup_{B \in \widehat{K} \backslash\{\widehat{B}\}} E^{B \cup \widehat{B}}\right)=\psi(F),
$$

completing the proof.

The following lemmas state some consequences of Anonymity and Monotonicity for the power index $\varphi$.

Lemma 4.3. Let $\varphi$ satisfy Monotonicity. Then $\varphi_{j}\left(U_{S}^{B}\right)=\varphi_{j}\left(U_{T}^{B}\right)$ for all $B \in P(A)$, all $S, T \in P(N)$ and all $j \in N \backslash(S \cup T)$.

Proof. Let $R \subseteq N$ and $j \in R, j \in N \backslash(S \cup T)$. Then

$$
\begin{aligned}
U_{S}^{B}(R) \backslash U_{S}^{B}(R \backslash\{j\}) & = \begin{cases}\{A\} & \text { if } R=\{j\} \\
\emptyset & \text { if } R \neq\{j\} \text { and } R \neq N \\
P_{0}(A) \backslash B^{+} & \text {if } R=N\end{cases} \\
& =U_{T}^{B}(R) \backslash U_{T}^{B}(R \backslash\{j\}) .
\end{aligned}
$$

Hence $\varphi_{j}\left(U_{S}^{B}\right)=\varphi_{j}\left(U_{T}^{B}\right)$ by monotonicity.

The proof of the following lemma is obvious and therefore omitted.

Lemma 4.4. Let $\varphi$ satisfy Anonymity. Then $\varphi_{i}\left(U_{S}^{B}\right)=\varphi_{j}\left(U_{T}^{B}\right)$ for all $B \in$ $P(A)$, all $S, T \in P(N)$ with $|S|=|T|$ and all $i \in S$ and $j \in T$.

A weight system is a collection $\omega=\left(\omega^{B}\right)_{B \in P_{0}(A)}$ of real numbers such that $\sum_{B \in P_{0}(A)} \omega^{B}=1$. Call a weight system $\omega$ non-negative if $\omega^{B} \geq 0$ for all $B \subseteq A$. For a weight system $\omega$ we define the power index $\Phi^{\omega}$ by

$$
\Phi^{\omega}(E)=\sum_{B \in P_{0}(A)} \omega^{B} S h\left(v_{B}^{E}\right)
$$

for every $E \in \mathcal{E}$. The main result of this section is as follows. 
Theorem 4.5. A power index $\varphi$ satisfies the Transfer Property, Anonymity, and Monotonicity if and only if there is a non-negative weight system $\omega$ such that $\varphi=\Phi^{\omega}$. Moreover, for any weight systems $\omega$ and $\omega^{\prime}, \Phi^{\omega}=\Phi^{\omega^{\prime}}$ implies $\omega=\omega^{\prime}$.

Proof. We leave it to the reader to verify that $\Phi^{\omega}$ satisfies the Transfer Property, Anonymity, and Monotonicity for any non-negative weight system $\omega$. Now let $\varphi$ be a power index with these three properties. By Lemma 4.2 it is sufficient to prove that there is a weight system $\omega$ as in the theorem such that $\varphi\left(U_{S}^{B}\right)=\Phi^{\omega}\left(U_{S}^{B}\right)$ for every $S \in P(N)$ and $B \in P(A)$.

Let $\omega$ be a weight system. If $S=N$ then $\varphi_{i}\left(U_{S}^{B}\right)=\Phi_{i}^{\omega}\left(U_{S}^{B}\right)=\frac{1}{n}$ for each $B \in P(A)$ and $i \in N$ by Anonymity of $\varphi$ and $\Phi^{\omega}$. For $S \in P(N) \backslash\{N\}$, $j \in N \backslash S$, and $B \in P(A)$ we have for $E=U_{S}^{B}$ :

$$
\begin{aligned}
\Phi_{j}^{\omega}\left(U_{S}^{B}\right) & =\sum_{B^{\prime} \in P_{0}(A)} \omega^{B^{\prime}} S h_{j}\left(v_{B^{\prime}}^{E}\right) \\
& =\sum_{B^{\prime} \in P_{0}(A), B^{\prime} \notin B^{+}} \omega^{B^{\prime}} S h_{j}\left(v_{B^{\prime}}^{E}\right)+\sum_{B^{\prime} \in P_{0}(A), B^{\prime} \in B^{+}} \omega^{B^{\prime}} S h_{j}\left(v_{B^{\prime}}^{E}\right) \\
& =\sum_{B^{\prime} \in P_{0}(A), B^{\prime} \notin B^{+}} \omega^{B^{\prime}} S h_{j}\left(v_{B^{\prime}}^{E}\right)+S h_{j}\left(v_{A}^{E}\right) \\
& =\sum_{B^{\prime} \in P_{0}(A), B^{\prime} \notin B^{+}} \frac{\omega^{B^{\prime}}}{n}+\frac{\omega^{A}}{n} .
\end{aligned}
$$

(Here we have used that $S h_{i}\left(v_{A}^{E}\right)=\frac{1}{n}$ for every $i \in N$, and that $S h_{j}\left(v_{B^{\prime}}^{E}\right)=0$ if $E=U_{S}^{B}, B \in P_{0}(A) \backslash\{A\}, B^{\prime} \in B^{+}$, and $j \in N \backslash S$.) On the other hand, by Lemma $4.3, \varphi_{j}\left(U_{S}^{B}\right)$ does not depend on $S$ (as long as $j \notin S$ ), and by Anonymity $\varphi\left(U_{S}^{B}\right)$ is completely determined by $\varphi_{j}\left(U_{S}^{B}\right)$. We now first show that the system of equations

$$
\varphi_{j}\left(U_{S}^{B}\right)=\sum_{B^{\prime} \in P_{0}(A), B^{\prime} \notin B^{+}} \frac{\omega^{B^{\prime}}}{n}+\frac{\omega^{A}}{n}, \quad B \in P_{0}(A)
$$

has a unique solution $\omega=\left(\omega^{B}\right)_{B \in P_{0}(A)}$. To show this, it is sufficient to show that the $\left(2^{|A|}-1\right) \times\left(2^{|A|}-1\right)$ matrix $M$ with rows and columns labelled by the sets in $P_{0}(A)$ and with elements $m_{B, C}$ defined by

$$
m_{B, C}= \begin{cases}1 & \text { if } B \neq A \text { and } C \notin\left(B^{+} \backslash\{A\}\right) \\ 1 & \text { if } B=A \text { or } C=A \\ 0 & \text { in all other cases }\end{cases}
$$

has full rank. We may order the rows of $M$ according to increasing size of $B$, that is, if $\left|B^{\prime}\right|<|B|$ then row $\left(m_{B, C}\right)_{C \in P_{0}(A)}$ is below row $\left(m_{B^{\prime}, C}\right)_{C \in P_{0}(A)}$. In particular, the last row corresponds to $A$. We also let the last column correspond to $A$, hence it has the form $\left(m_{B, A}\right)_{B \in P_{0}(A)}$. We fix the other columns by letting 
the diagonal elements, except the last one, be of the form $m_{B, A \backslash B}$ (the last one is $\left.m_{A, A}\right)$. From these assumptions it follows, in particular, that $B \cap C \neq \emptyset$ whenever $m_{B, C}$ is below the diagonal.

Next, we manipulate the rows of $M$ by letting, for all $B, C \in P_{0}(A)$,

$$
\widetilde{m}_{B, C}=m_{B, C}+\sum_{\emptyset \neq B^{\prime} \subsetneq B}(-1)^{|B|-\left|B^{\prime}\right|} m_{B^{\prime}, C} .
$$

This way we obtain the matrix $\widetilde{M}$, and it is sufficient to prove that $\widetilde{M}$ has full rank. We consider three different types of elements $m_{B, C}$ of the original matrix $M$.

(a) Suppose $m_{B, C}=0$. Then $B, C \neq A$ and $B \subseteq C$ and hence $m_{B^{\prime}, C}=0$ for all $\emptyset \neq B^{\prime} \subsetneq B$, so that $\widetilde{m}_{B, C}=m_{B, C}=0$.

(b) Suppose $m_{B, C}=1$ and $m_{B, C}$ is below the diagonal of $M$. Then $B \cap(A \backslash$ $C) \neq \emptyset$ and $B \cap C \neq \emptyset$. Now

$$
\begin{aligned}
\widetilde{m}_{B, C} & =m_{B, C}+\sum_{\emptyset \neq B^{\prime} \subsetneq B}(-1)^{|B|-\left|B^{\prime}\right|} m_{B^{\prime}, C} \\
& =m_{B, C}+\sum_{\emptyset \neq B^{\prime} \subsetneq B}(-1)^{|B|-\left|B^{\prime}\right|}-\sum_{\emptyset \neq B^{\prime} \subsetneq B, B^{\prime} \subseteq C}(-1)^{|B|-\left|B^{\prime}\right|} \\
& =m_{B, C}+\sum_{B^{\prime} \subsetneq B}(-1)^{|B|-\left|B^{\prime}\right|}-\sum_{B^{\prime} \subsetneq B, B^{\prime} \subseteq C}(-1)^{|B|-\left|B^{\prime}\right|} \\
& =1+(-1)-\sum_{B^{\prime} \subseteq B \cap C}(-1)^{|B|-\left|B^{\prime}\right|} \\
& =1+(-1)+0 .
\end{aligned}
$$

In the fourth equality we have used the fact that $B \cap C \neq \emptyset$. (Furthermore, we have used that $\sum_{k=0}^{\ell}(-1)^{k}\left(\begin{array}{l}\ell \\ k\end{array}\right)=0$.)

(c) Finally, consider a diagonal element $m_{B, A \backslash B}$ with $B \neq A$. Then

$$
\begin{aligned}
\tilde{m}_{B, A \backslash B} & =m_{B, A \backslash B}+\sum_{\emptyset \neq B^{\prime} \subsetneq B}(-1)^{|B|-\left|B^{\prime}\right|} m_{B^{\prime}, A \backslash B} \\
& =1+\sum_{\emptyset \neq B^{\prime} \subsetneq B}(-1)^{|B|-\left|B^{\prime}\right|} \\
& =1+0-(-1)^{|B|}-(-1)^{0} \\
& = \pm 1 .
\end{aligned}
$$

In particular, $\widetilde{m}_{B, A \backslash B} \neq 0$ for every $B \in P_{0}(A) \backslash\{A\}$. By exactly the same argument (replace $A \backslash B$ by $A$ ) it follows that $\widetilde{m}_{A, A} \neq 0$.

From (a)-(c) we conclude that $\widetilde{M}$ is an upper triangular matrix with non-zero diagonal elements and thus has full rank. Therefore, the system of equations 
(4) has a unique solution $\omega=\left(\omega^{B}\right)_{B \in P_{0}(A)}$. We next show that the numbers $\omega_{B}$ sum to 1 , as follows. For every $i \in N$, by $(3), \varphi_{i}\left(U_{\emptyset}^{A}\right)=\sum_{B \in P_{0}(A)} \frac{\omega^{B}}{n}$, and by summing over $i \in N$ we obtain $n \sum_{B \in P_{0}(A)} \frac{\omega^{B}}{n}=1$.

Non-negativity of $\omega$ is implied by Monotonicity of $\varphi$ as follows. Suppose, to the contrary, that $\omega^{B}<0$ for some $B \in P_{0}(A)$. Let $S \in P_{0}(N) \backslash\{N\}$ and let $i \in N \backslash S$. Let $E=\bigcup_{B^{\prime} \nsupseteq B} U_{S}^{B^{\prime}}$ and let $F=U_{S}^{B}$. Then $E, F \in \mathcal{E}$, and for $T \subseteq N$ with $i \in T$,

$$
E(T) \backslash E(T \backslash\{i\})= \begin{cases}\{A\} & \text { if } T=\{i\} \\ \left(P_{0}(A) \backslash B^{+}\right) \cup\{B\} & \text { if } T=N \\ \emptyset & \text { otherwise }\end{cases}
$$

and

$$
F(T) \backslash F(T \backslash\{i\})= \begin{cases}\{A\} & \text { if } T=\{i\} \\ P_{0}(A) \backslash B^{+} & \text {if } T=N \\ \emptyset & \text { otherwise. }\end{cases}
$$

Therefore Monotonicity of $\varphi$ implies $\varphi_{i}(F) \leq \varphi_{i}(E)$. On the other hand, by (4),

$$
\varphi_{i}(F)=\sum_{B^{\prime} \in P_{0}(A): B^{\prime} \notin B^{+}} \frac{\omega^{B^{\prime}}}{n}+\frac{\omega^{A}}{n}
$$

and

$$
\begin{aligned}
\varphi_{i}(E)= & \Phi^{\omega}(E) \\
= & \sum_{B^{\prime} \in P_{0}(A)} \omega^{B^{\prime}} S h_{i}\left(v_{B^{\prime}}^{E}\right) \\
= & \sum_{B^{\prime} \in P_{0}(A): B^{\prime} \notin B^{+}} \omega^{B^{\prime}} S h_{i}\left(v_{B^{\prime}}^{E}\right)+\sum_{B^{\prime} \in P_{0}(A): B^{\prime} \in B^{+}, B^{\prime} \neq B} \omega^{B^{\prime}} S h_{i}\left(v_{B^{\prime}}^{E}\right) \\
& \quad+\omega^{B} S h_{i}\left(v_{B}^{E}\right) \\
= & \sum_{B^{\prime} \in P_{0}(A): B^{\prime} \notin B^{+}} \frac{\omega^{B^{\prime}}}{n}+\frac{\omega^{A}}{n}+\frac{\omega^{B}}{n} \\
< & \varphi_{i}(F)
\end{aligned}
$$

which is a contradiction. Hence, $\omega$ is non-negative. The last claim in the theorem follows by unicity of $\omega$ as proved above.

In the remainder of this section we consider further properties of power indices of the form $\Phi^{\omega}$. 


\subsection{Connection with simple games}

With a simple game $v$ we can associate an effectivity function $E^{v}$ in a natural way by defining

$$
E^{v}(S)= \begin{cases}P_{0}(A) & \text { if } v(S)=1 \\ \{A\} & \text { if } v(S)=0 \text { and } S \neq \emptyset \\ \emptyset & \text { if } S=\emptyset .\end{cases}
$$

Then $v_{B}^{E^{v}}=v$ for every $B \in P_{0}(A)$. From this the following result is straightforward.

Theorem 4.6. For every weight system $\omega$ and every simple game $v, \Phi^{\omega}\left(E^{v}\right)=$ $S h(v)$.

Thus, for effectivity functions associated with simple games, $\Phi^{\omega}$ reduces to the Shapley value for every weight system $\omega$.

\subsection{Dual games and polar effectivity functions}

For a simple game $v$, the dual game is the game $v^{*}$ defined by $v^{*}(S)=v(N)-$ $v(N \backslash S)$ for all $S \in P(N)$. Note that $v^{*}$ is again a simple game (in particular, it is monotonic).

For an effectivity function $E \in \mathcal{E}$, the polar effectivity function is the effectivity function $E^{*}$ defined by $E^{*}(\emptyset)=\emptyset$ and $E^{*}(S)=\left\{B \in P_{0}(A): B \cap B^{\prime} \neq\right.$ $\emptyset$ for all $\left.B^{\prime} \in E(N \backslash S)\right\}$. Note that $E^{*} \in \mathcal{E}$.

The following results are easily established.

Theorem 4.7. Let $E \in \mathcal{E}$. Then:

(a) $\left(v_{B}^{E}\right)^{*}=v_{A \backslash B}^{E^{*}}$ for all $B \in P_{0}(A) \backslash\{A\}$.

(b) $\left(v_{A}^{E}\right)^{*}=v_{B}^{F^{*}}$ for all $B \in P_{0}(A) \backslash\{A\}$, where $F \in \mathcal{E}$ is defined by $F(\emptyset)=\emptyset$ and $F(S)=P_{0}(A)$ for all $S \in P_{0}(N)$.

For a weight system $\omega$ define the weight system $\bar{\omega}$ by $\bar{\omega}^{B}=\omega^{A \backslash B}$ for all $B \in P_{0}(A) \backslash\{A\}$ and $\bar{\omega}^{A}=\omega^{A}$. Then we have the following consequence of Theorem 4.7.

Theorem 4.8. $\Phi^{\omega}(E)=\Phi^{\bar{\omega}}\left(E^{*}\right)$ for every $E \in \mathcal{E}$ and every weight system $\omega$. In particular, $\Phi^{\omega}(E)=\Phi^{\omega}\left(E^{*}\right)$ if $\omega^{B}=\omega^{A \backslash B}$ for all $B \in P_{0}(A) \backslash\{A\}$.

If an effectivity function is interpreted as describing what coalitions can guarantee on their own, then the polar effectivity function describes what a coalition cannot be kept from by its complement. Theorem 4.8 says that the power of a player in an effectivity function is the same in its polar if we switch the weights of sets of alternatives with those of their complements, in line with these interpretations. 


\subsection{Neutrality}

For a permutation $\pi$ of $A$ and $E \in \mathcal{E}$, define $E_{\pi} \in \mathcal{E}$ by $E_{\pi}(S)=\{\pi(B): B \in$ $E(S)\}$.

Neutrality $\varphi(E)=\varphi\left(E_{\pi}\right)$ for every permutation $\pi$ of $A$ and every $E \in \mathcal{E}$.

We omit the easy proof of the following result.

Theorem 4.9. A power index $\varphi$ satisfies the Transfer Property, Anonymity, Monotonicity, and Neutrality if and only if there is a non-negative weight system $\omega$ with $\omega^{B}=\omega^{B^{\prime}}$ for all $B, B^{\prime} \in P_{0}(A)$ with $|B|=\left|B^{\prime}\right|$, such that $\varphi=\Phi^{\omega}$.

\subsection{Strong monotonicity}

The premise of Strong Monotonicity is much weaker than the one of Monotonicity: we are now comparing only a player's (marginal) ability to enforce (single) outcomes. As may be expected intuitively, for a power index $\Phi^{\omega}$ this condition results in the weights of non-singleton but strict subsets of alternatives becoming zero.

Theorem 4.10. Let $A$ be a finite set. A power index $\varphi$ satisfies the Transfer Property, Anonymity, and Strong Monotonicity if and only if there is a nonnegative weight system $\omega$ with $\omega^{B}=0$ for all $B \in P(A) \backslash\{A\}$ with $|B| \geq 2$, such that $\varphi=\Phi^{\omega}$.

Proof. Showing that a power index $\Phi^{\omega}$ as in the statement of the theorem satisfies Strong Monotonicity (all the other axioms are satisfied by Theorem $4.5)$ is straightforward and omitted here. For the only-if direction, again by Theorem 4.5, for a power index $\varphi$ satisfying the three conditions there is a weight system $\omega$ such that $\varphi=\Phi^{\omega}$. For $B \in P(A) \backslash\{A\}$ with $|B| \geq 2, i \in N$, $S \in P_{0}(N)$, and $a \in A$ we have that $\{a\} \in U_{S}^{B}(T) \backslash U_{S}^{B}(T \backslash\{i\})$ if and only if $T=N$. This is the case if and only if $\{a\} \in U_{N}^{B}(T) \backslash U_{N}^{B}(T \backslash\{i\})$. Hence, by Strong Monotonicity, $\Phi_{i}^{\omega}\left(U_{S}^{B}\right)=\Phi_{i}^{\omega}\left(U_{N}^{B}\right)=\frac{1}{n}$ for all $S \in P_{0}(N), i \in N$, and $B \in P(A) \backslash\{A\}$ with $|B| \geq 2$. By (4) it then follows that $\omega^{B}=0$ for all such $B$.

The next example provides an application of a strongly monotonic power index to US legislation.

Example 4.11 (Example 2.5 continued). Recall that the Republicans have simple majorities both in the Senate and in the House. This means that for $N=\{R, D\}$, no singleton is effective for any $\{a\}$ with $a \in A_{1} \cup A_{2} \cup A_{4}$, but $\{D\}$ is effective for any $\{a\}$ with $a \in A_{3}$. Hence, as long as no party has a two third majority in both chambers of Congress, the President's party is more powerful according to a power index in Theorem 4.10 . 


\subsection{Null players}

If we require that a power index of the form $\Phi^{\omega}$ additionally satisfies the Null Player Property, then it must be the case that $\omega_{A}=0$, since the simple game $v_{A}^{E}$ is the only game in the definition of $\Phi^{\omega}$ in which a null player in $E$ would get a positive amount, namely $\frac{1}{n}$. This insight results in obvious corollaries of Theorems $4.5-4.10$ by adding the Null player Property. In the next section the Null Player axiom will play a more prominent role.

\section{$5 \quad$ Infinitely many alternatives}

In this section we assume that $A$ is a possibly infinite set, endowed with a topology. More precisely, we assume that $A$ is a $T_{1}$-space, i.e. each set $\{a\}$ with $a \in A$ is closed. ${ }^{1}$ The set of all nonempty closed subsets of $A$ is denoted by $\mathcal{T}$. An effectivity function for $\mathcal{T}$ is defined as before, i.e. as in Definition 2.1. ${ }^{2}$

The set of monotonic and superadditive effectivity functions $\mathcal{E}$ is closed in the following sense. ${ }^{3}$

Lemma 5.1. Let $\left(E_{k}\right)_{k \in \mathbb{N}}$ be a sequence in $\mathcal{E}$ with $E_{1} \subseteq E_{2} \subseteq$... Then $\bigcup_{k \in \mathbb{N}} E_{k} \in \mathcal{E}$.

Proof. Let $E=\bigcup_{k \in \mathbb{N}} E_{k}$. Then $E(S)=\bigcup_{k \in \mathbb{N}} E_{k}(S) \subseteq \mathcal{T}$ since $E_{k}(S) \subseteq \mathcal{T}$ for all $k \in \mathbb{N}$. Further $E(\emptyset)=\emptyset, A \in E(S)$ for all $S \subseteq N$ and $E(N)=\mathcal{T}$. Hence, $E$ is a topological effectivity function for $\mathcal{T}$. Further, if $T \subseteq S, B, B^{\prime} \in \mathcal{T}$ with $B \subseteq B^{\prime}$ and $B \in E(T)$, then there is $k \in \mathbb{N}$ with $B \in E_{k}(T)$ and therefore $B^{\prime} \in E_{k}(S)$ by the monotonicity of $E_{k}$. Hence, $B \in E(S)$, i.e. $E$ is monotonic. Finally, let $B, B^{\prime} \in \mathcal{T}$ and let $S, T \subseteq N$ be nonempty and disjoint such that $B \in E(T)$ and $B^{\prime} \in E(S)$. Then there are $k, m \in \mathbb{N}$ such that $B \in E_{k}(T)$ and $B^{\prime} \in E_{m}(S)$. In particular, $B \in E_{l}(T)$ and $B^{\prime} \in E_{l}(S)$ for $l=\max \{k, m\}$. Hence, $B \cap B^{\prime} \in E_{l}(S \cup T) \subseteq E(S \cup T)$, i.e. $E$ is superadditive.

For an effectivity function $E$ and $S \in P_{0}(N)$ let $E_{S}$ be defined by

$$
E_{S}(T)= \begin{cases}\mathcal{T} & \text { if } T=N \\ E(S) & \text { if } T \supseteq S \text { and } T \neq N \\ \{A\} & \text { if } T \nsupseteq S \text { and } T \neq \emptyset \\ \emptyset & \text { if } T=\emptyset .\end{cases}
$$

Then $E_{S} \in \mathcal{E}$ for every $E \in \mathcal{E}$ and $S \in P_{0}(N)$. An effectivity function $E \in \mathcal{E}$ may be decomposed in way that is related to the one in Lemma 4.1.

Lemma 5.2. Let $E \in \mathcal{E}$ and let $I \subseteq P_{0}(N)$ such that $S^{\prime} \in I$ whenever there is $S \in I$ with $S \subseteq S^{\prime}$, and let $F_{I}=\bigcup_{S \in I} E_{S}$. Then $F_{I} \in \mathcal{E}$.

\footnotetext{
${ }^{1}$ Typical examples are topologies that are derived from a metric on $A$.

${ }^{2}$ Such an effectivity function is also sometimes called topological.

${ }^{3}$ Recall that for $E \in \mathcal{E}$ and $S \in P_{0}(N), E(S)$ is a collection of closed sets, and does not need to be closed itself.
} 
Proof. Clearly, $F_{I}$ is an effectivity function. Let $S \subseteq T$ and let $B \in F_{I}(S)$ and $B^{\prime} \in \mathcal{T}$ with $B \subseteq B^{\prime}$. Then there is $S^{\prime} \in I$ such that $B \in E_{S^{\prime}}(S)$. Hence, $B^{\prime} \in E_{S^{\prime}}(T)$ by the monotonicity of $E_{S^{\prime}}$, and therefore $B^{\prime} \in F_{I}(T)$. For superadditivity, let $B, B^{\prime} \in \mathcal{T} \backslash\{A\}$ (if either of these sets equals $A$, there is nothing to show) and let $S, T \subseteq N$ be nonempty and disjoint such that $B \in F_{I}(S)$ and $B^{\prime} \in F_{I}(T)$. Then there are $S^{\prime}, T^{\prime} \in I$ such that $B \in E_{S^{\prime}}(S) \subseteq$ $E(S)$ and $B^{\prime} \in E_{T^{\prime}}(T) \subseteq E(T)$. In particular, since $B, B^{\prime} \neq A$, it must holds that $S^{\prime} \subseteq S$ and $T^{\prime} \subseteq T$. Hence, $B \cap B^{\prime} \in E\left(S^{\prime} \cup T^{\prime}\right) \subseteq E(S \cup T)$ by the superadditivity of $E$. Since $B \cap B^{\prime} \in E_{S \cup T}(S \cup T)$, and since $S \cup T \in I$, it holds that $B \cap B^{\prime} \in F_{I}(S \cup T)$.

This lemma implies the following one which is quite in the spirit of Lemma 4.2.

Lemma 5.3. Let $\varphi$ and $\psi$ be power indices satisfying the Transfer Property and such that $\varphi\left(E_{S}\right)=\psi\left(E_{S}\right)$ for all $E \in \mathcal{E}$ and all $S \subseteq N$. Then $\varphi=\psi$.

Proof. For any effectivity function $E$ there is a set $I_{E} \subseteq P_{0}(N)$ with $S^{\prime} \in I_{E}$ whenever there is $S \in I_{E}$ with $S \subseteq S^{\prime}$, such that $E=F_{I_{E}}$ (with $F_{I_{E}}$ defined as in Lemma 5.2). If $\left|I_{E}\right|=1$, i.e. $E=E_{S}$ for some $S$, then $\varphi(E)=\psi(E)$ by assumption. Let $\left|I_{E}\right| \geq 2$ and let the claim be true for all effectivity functions $E^{\prime}$ with $\left|I_{E^{\prime}}\right|<\left|I_{E}\right|$. Let $S \in I_{E}$ be minimal. Then

$$
E_{S} \cap\left(\bigcup_{T \in I_{E} \backslash\{S\}} E_{T}\right)=\bigcup_{T \in I_{E} \backslash\{S\}}\left(E_{T} \cap E_{S}\right)=\bigcup_{T \in I_{E} \backslash\{S\}} E_{T}^{\prime}
$$

where $E^{\prime}=E \cap E_{S}$ is a monotonic and superadditive effectivity function by Lemma 2.2. The set

$$
\left\{T: T \in I_{E} \backslash\{S\}\right\}
$$

satisfies the condition of Lemma 5.2, hence $\cup_{T \in I_{E} \backslash\{S\}} E_{T}^{\prime} \in \mathcal{E}$. This means that the Transfer Property applies and

$$
\begin{aligned}
\varphi\left(\bigcup_{T \in I_{E}} E_{T}\right) & =\varphi\left(E_{S} \cup\left(\bigcup_{T \in I_{E} \backslash\{S\}} E_{T}\right)\right) \\
& =\varphi\left(E_{S}\right)+\varphi\left(\bigcup_{T \in I_{E} \backslash\{S\}} E_{T}\right)-\varphi\left(\bigcup_{T \in I_{E} \backslash\{S\}} E_{T}^{\prime}\right) \\
& =\psi\left(E_{S}\right)+\psi\left(\bigcup_{T \in I_{E} \backslash\{S\}} E_{T}\right)-\psi\left(\bigcup_{T \in I_{E} \backslash\{S\}} E_{T}^{\prime}\right) \\
& =\psi\left(\bigcup_{T \in I_{E}} E_{T}\right) .
\end{aligned}
$$

by the induction hypothesis. 
We introduce the following regularity condition.

Continuity For any sequence $\left(E_{k}\right)_{k \in \mathbb{N}}$ of effectivity functions with $E_{1} \subseteq E_{2} \subseteq$ $\ldots$ it holds that

$$
\varphi\left(\bigcup_{k=1}^{\infty} E_{k}\right)=\lim _{k \rightarrow \infty} \varphi\left(E_{k}\right) .
$$

Note that the left hand side is well defined by Lemma 5.1. In case of a finite set $A$, continuity is trivially satisfied by any power index.

For a probability measure $\mu$ on $P(A)$ we define the map $\Phi^{\mu}$ on $\mathcal{E}$ by

$$
\Phi_{i}^{\mu}(E)=\int_{A} S h_{i}\left(v_{\{a\}}^{E}\right) d \mu(a)
$$

for every $E \in \mathcal{E}$ and $i \in N$. Clearly, $\sum_{i \in N} \Phi_{i}^{\mu}(E)=1$ for every $E \in \mathcal{E}(\mathcal{T})$, so that $\Phi^{\mu}$ is a power index. ${ }^{4}$

Theorem 5.4. Let $\varphi$ be a power index. Then $\varphi$ satisfies the Transfer Property, Anonymity, Strong Monotonicity, Continuity, and the Null Player Property if and only if there is a probability measure $\mu$ such that $\varphi=\Phi^{\mu}$.

Proof. First, let $\mu$ be a probability measure on $P(A)$. We show that $\Phi^{\mu}$ satisfies the five properties in the theorem. It is straightforward that $\Phi^{\mu}$ satisfies Anonymity; and since a null player in $E \in \mathcal{E}$ obtains 0 in every simple game $v_{\{a\}}^{E}, \Phi^{\mu}$ satisfies the Null Player Property. If $E, F \in \mathcal{E}$ and $i \in N$ satisfy the premise of Strong Monotonicity, then $S h_{i}\left(v_{\{a\}}^{E}\right) \leq S h_{i}\left(v_{\{a\}}^{F}\right)$ for all $a \in A$. Hence, $\Phi^{\mu}$ satisfies Strong Monotonicity.

To see that $\Phi^{\mu}$ satisfies the Transfer Property, let $E, F \in \mathcal{E}$ with $E \cup F \in \mathcal{E}(\mathcal{T})$. Then

$$
\begin{aligned}
\Phi^{\mu}(E \cup F) & =\int_{A} S h_{i}\left(v_{\{a\}}^{E \cup F}\right) d \mu(a)=\int_{A} S h_{i}\left(v_{\{a\}}^{E} \vee v_{\{a\}}^{F}\right) d \mu(a) \\
& =\int_{A}\left(S h_{i}\left(v_{\{a\}}^{E}\right)+S h_{i}\left(v_{\{a\}}^{F}\right)-S h_{i}\left(v_{\{a\}}^{E} \wedge v_{\{a\}}^{F}\right)\right) d \mu(a) \\
& =\Phi^{\mu}(E)+\Phi^{\mu}(F)-\Phi^{\omega}(E \cap F),
\end{aligned}
$$

where the second equality follows from Lemma 2.4 and the third one from the Transfer Property of the Shapley value Dubey (1975).

For Continuity let $\left(E_{k}\right)_{k \in \mathbb{N}} \subseteq \mathcal{E}$ be an ascending sequence of effectivity functions. Then for any $S \subseteq N$ and any $a \in A$ it holds that $\{a\} \in \bigcup_{k=1}^{\infty} E_{k}(S)$ if and only

\footnotetext{
${ }^{4}$ Unless stated otherwise, we assume that probability measures are defined on the $\sigma$-field $P(A)$, i.e., the power set of $A$. Note, however, that all results in this section are also valid for probability measures defined on the $\sigma$-field of Borel sets - i.e., the $\sigma$-field generated by the topology on $A$.
} 
if $v_{a}^{\bigcup_{k=1}^{\infty} E_{k}}(S)=1$, or equivalently $\lim _{k \rightarrow \infty} v_{a}^{E_{k}}(S)=1$. Therefore

$$
\begin{aligned}
\Phi_{i}^{\mu}\left(\bigcup_{k=1}^{\infty} E_{k}\right) & =\int_{A} S h_{i}\left(v_{a}^{\bigcup_{k=1}^{\infty} E_{k}}\right) d \mu(a)=\int_{A} S h_{i}\left(\lim _{k \rightarrow \infty} v_{a}^{E_{k}}\right) d \mu(a) \\
& =\int_{A} \lim _{k \rightarrow \infty} S h_{i}\left(v_{a}^{E_{k}}\right) d \mu(a)=\lim _{k \rightarrow \infty} \Phi_{i}^{\mu}\left(E_{k}\right)
\end{aligned}
$$

by the Lebesgue Dominated Convergence Theorem as for all $a \in A$ the integrand $S h_{i}\left(v_{a}^{E_{k}}\right)$ is constant for sufficiently large $k$.

Suppose now that $\varphi$ is a power index with the five properties in the theorem, let $S \in P_{0}(N)$, let $i \in N \backslash S$, and define a function $\mu$ on $P(A)$ by

$$
\mu(B)=1-n \varphi_{i}\left(E^{0} \cup\left(\bigcup_{b \in B} U_{S}^{\{b\}}\right)\right)
$$

for all $B \in P(A)$, where $E^{0}$ is the effectivity function with $E(S)=\{A\}$ for all $S \neq \emptyset, S \neq N$. Note that $\mu$ is well defined since $E^{0} \cup\left(\bigcup_{b \in B} U_{S}^{\{b\}}\right) \in \mathcal{E}$ and since, by Anonymity and Strong Monotonicity, the right hand side does not depend on $S \in P_{0}(N)$ and $i \in N$, provided $i \notin S$. We first show that $\mu$ is a probability measure. Clearly,

$$
\mu(\emptyset)=1-n \varphi_{i}\left(E^{0}\right)=1-n \frac{1}{n}=0,
$$

where the second equality follows by Anonymity. By Strong Monotonicity, $\varphi_{i}\left(E^{0} \cup \bigcup_{b \in B} U_{S}^{\{b\}}\right) \leq \varphi_{i}\left(E^{0}\right)$ for all $i \in N \backslash S$ and all $B \in P(A)$. Hence,

$$
\mu(B) \geq 1-n \varphi_{i}\left(E^{0}\right)=0 .
$$

Also, $\mu(A)=1-n \varphi_{i}\left(\bigcup_{b \in A} U_{S}^{\{b\}}\right)=1$ since $i \notin S$ is a null player in $\bigcup_{b \in A} U_{S}^{\{b\}}$. Further, for any two disjoint sets $B, C \subseteq A$ it holds that

$$
\begin{aligned}
\mu(B \cup C) & =1-n \varphi_{i}\left(\bigcup_{b \in B \cup C} U_{S}^{\{b\}}\right) \\
& =1-n\left(\varphi_{i}\left(\bigcup_{b \in B} U_{S}^{\{b\}}\right)+\varphi_{i}\left(\bigcup_{b \in C} U_{S}^{\{b\}}\right)-\varphi_{i}\left(\bigcup_{b \in B} \bigcup_{c \in C} U_{S}^{\{b, c\}}\right)\right) \\
& =1-n \varphi_{i}\left(\bigcup_{b \in B} U_{S}^{\{b\}}\right)-n \varphi_{i}\left(\bigcup_{b \in C} U_{S}^{\{b\}}\right)+n \frac{1}{n} \\
& =\mu(B)+\mu(C),
\end{aligned}
$$

where the second equality follows from the Transfer Property and the third equality follows from Strong Monotonicity applied to $\bigcup_{b \in B} \bigcup_{c \in C} U_{S}^{\{b, c\}}$ and 
$E^{0}$. Hence, $\mu$ is finitely additive. Further, for any sequence $\left(B_{k}\right)_{k \in \mathbb{N}}$ with $B_{1} \subseteq B_{2} \subseteq \ldots$ it holds that

$$
\begin{aligned}
\mu\left(\bigcup_{k \in \mathbb{N}} B_{k}\right) & =1-n \varphi_{i}\left(\bigcup_{k \in \mathbb{N}}\left(\bigcup_{b \in B_{k}} U_{S}^{\{b\}}\right)\right) \\
& =1-n \lim _{k \rightarrow \infty} \varphi_{i}\left(\bigcup_{b \in B_{k}} U_{S}^{\{b\}}\right) \\
& =\lim _{k \rightarrow \infty} \mu\left(B_{k}\right) .
\end{aligned}
$$

This shows that $\mu$ is continuous from below, which together with finite additivity implies that $\mu$ is $\sigma$-additive. Hence, $\mu$ is a probability measure.

In order to show that $\varphi$ and $\Phi^{\mu}$ coincide, note that for $S \in P_{0}(N)$ and $i \notin S$ we have

$$
\begin{aligned}
\varphi_{i}\left(\bigcup_{b \in B} U_{S}^{\{b\}}\right) & =\frac{1}{n}(1-\mu(B))=\frac{1}{n} \mu(A \backslash B) \\
& =\int_{A \backslash B} S h_{i}\left(v_{a}^{\cup_{b \in B} U_{S}^{\{b\}}}\right) d \mu(a)=\int_{A} S h_{i}\left(v_{a}^{\cup_{b \in B} U_{S}^{\{b\}}}\right) d \mu(a) \\
& =\Phi_{i}^{\mu}\left(\bigcup_{b \in B} U_{S}^{\{b\}}\right),
\end{aligned}
$$

where the third equality follows from the fact that $S h_{i}\left(v_{a}^{\cup_{b \in B} U_{S}^{\{b\}}}\right)=\frac{1}{n}$ for $a \in A \backslash B$; the fourth equality follows since $S h_{i}\left(v_{a}^{\cup_{b \in B} U_{S}^{\{b\}}}\right)=0$ for $a \in B$ as $i \notin S$; and the last equality follows by definition of $\Phi^{\mu}$.

Let $E \in \mathcal{E}$. For $S \in P_{0}(N)$, we have that

$$
\begin{aligned}
\varphi\left(E_{S}\right) & =\varphi\left(\bigcup_{B \in E(S)} U_{S}^{B}\right)=\varphi\left(\bigcup_{B \in E(S):|B|=1} U_{S}^{B}\right) \\
& =\Phi^{\mu}\left(\bigcup_{B \in E(S):|B|=1} U_{S}^{B}\right)=\Phi^{\mu}\left(E_{S}\right)
\end{aligned}
$$

where the second and the last equality follow from Strong Monotonicity. By Lemma $5.3 \varphi$ and $\Phi^{\mu}$ coincide.

Example 5.5 (Example 2.5 continued). We apply the power index in Theorem 5.4 to the legislative process from the introduction. ${ }^{5}$ Clearly, $S h_{p}\left(v_{\{a\}}^{E}\right)=1$ for

\footnotetext{
${ }^{5}$ Since strictly speaking the number of alternatives is finite here, this is also an application of the results of Section 4, in particular 4.5.
} 
all $a \in A_{3}$. For $a \in A_{1}$ we have that all $h \in H$ are null players and hence

$$
\begin{aligned}
& S h_{s}\left(v_{\{a\}}^{E}\right)=\frac{33 ! 67 !}{101 !}\left(\begin{array}{c}
99 \\
66
\end{array}\right) \approx 6.634 \times 10^{-3}, \\
& S h_{p}\left(v_{\{a\}}^{E}\right) \approx 0.3366 .
\end{aligned}
$$

For $a \in A_{2}$ we have

$$
\begin{aligned}
& S h_{s}\left(v_{\{a\}}^{E}\right)=\left(\begin{array}{c}
99 \\
50
\end{array}\right) \sum_{k=218}^{435}(52+k)^{-1}\left(\begin{array}{c}
536 \\
52+k
\end{array}\right)^{-1}\left(\begin{array}{c}
435 \\
k
\end{array}\right) \approx 2.884 \times 10^{-3}, \\
& S h_{h}\left(v_{\{a\}}^{E}\right)=\left(\begin{array}{c}
434 \\
217
\end{array}\right) \sum_{k=51}^{100}(219+k)^{-1}\left(\begin{array}{c}
536 \\
219+k
\end{array}\right)^{-1}\left(\begin{array}{c}
100 \\
k
\end{array}\right) \approx 5.429 \times 10^{-4}, \\
& S h_{p}\left(v_{\{a\}}^{E}\right) \approx 0.4754 .
\end{aligned}
$$

For $a \in A_{4}$ we have

$$
\begin{aligned}
S h_{s}\left(v_{\{a\}}^{E}\right)= & \left(\begin{array}{c}
99 \\
50
\end{array}\right) \sum_{k=218}^{435}(52+k)^{-1}\left(\begin{array}{c}
536 \\
52+k
\end{array}\right)^{-1}\left(\begin{array}{c}
435 \\
k
\end{array}\right) \\
& +\left(\begin{array}{c}
99 \\
66
\end{array}\right) \sum_{k=290}^{435}(67+k)^{-1}\left(\begin{array}{c}
536 \\
67+k
\end{array}\right)^{-1}\left(\begin{array}{c}
435 \\
k
\end{array}\right) \\
\approx & 4.374 \times 10^{-3} \\
S h_{p}\left(v_{\{a\}}^{E}\right)= & \left(\begin{array}{c}
434 \\
217
\end{array}\right) \sum_{k=51}^{100}(219+k)^{-1}\left(\begin{array}{c}
536 \\
219+k
\end{array}\right)^{-1}\left(\begin{array}{c}
100 \\
k
\end{array}\right) \\
& +\left(\begin{array}{c}
435 \\
289
\end{array}\right) \sum_{k=67}^{100}(290+k)^{-1}\left(\begin{array}{c}
536 \\
290+k
\end{array}\right)^{-1}\left(\begin{array}{c}
100 \\
k
\end{array}\right) \\
\approx & 8.244 \times 10^{-4} \\
S h_{p}\left(v_{\{a\}}^{E}\right) \approx & 0.204 .
\end{aligned}
$$

For the overall legislative process we therefore find

$$
\begin{aligned}
\varphi_{s}(E) & =\frac{1}{1000}\left(6.634 \mu\left(A_{1}\right)+2.884 \mu\left(A_{2}\right)+4.374 \mu\left(A_{4}\right)\right) \\
\varphi_{h}(E) & =\frac{1}{10000}\left(5.429 \mu\left(A_{2}\right)+8.244 \mu\left(A_{4}\right)\right) \\
\varphi_{p}(E) & =\frac{1}{10}\left(3.366 \mu\left(A_{1}\right)+4.754 \mu\left(A_{2}\right)+\mu\left(A_{3}\right)+2.04 \mu\left(A_{4}\right)\right) .
\end{aligned}
$$




\subsection{An application of the infinite case: a spatial power index}

The Shapley value (Shapley, 1953b) can be used as a measure of power of the players in a simple game, where this simple game represents for instance the political situation in a democratic parliament. In this case the Shapley value is also called Shapley-Shubik power index (Shapley and Shubik, 1954). It sets the power of a player equal to the probability that this player is pivotal (makes a losing coalition winning) if coalitions are formed in a random order. This way of measuring power, however, takes neither the issues at stake nor the positions of the players (political parties) into account. Clearly, our approach improves on this, as is exemplified by the case of US legislation above.

Political scientists often consider spatial models to remedy this defect (e.g. Enelow and Hinich, 1984, 1990; Grofman et al., 1987). A well-known adaptation of the Shapley value to the spatial context is the Owen-Shapley spatial power index (Owen and Shapley, 1989; Martin et al., 2014; Peters and Zarzuelo, 2016).

Here, we present a spatial power index, in the spirit of the Owen-Shapley spatial power index, which is a power index of a topological effectivity function as in Theorem 5.4. With some modification of our model also the Owen-Shapley spatial power index can be obtained as a special case of a power index in this theorem, as we will explain below (Remark 5.7).

As before, $N=\{1, \ldots, n\}$ is the set of players. A simple game $v$ (for $N$ ) is proper if $v(S)=1$ implies that $v(N \backslash S)=0$ for each $S \in P(N)$. Let $k \in \mathbb{N}$, $k \geq 2$. A spatial game is a pair $g=(v, p)$ where $v$ is a proper simple game and $p=\left(p^{1}, \ldots, p^{n}\right) \in\left(\mathbb{R}^{k}\right)^{N}$ with $p^{i} \neq p^{j}$ whenever $i \neq j$, for all $i, j \in N$. Here, $p^{i} \in \mathbb{R}^{k}$ is the position of player $i$. Following Owen and Shapley (1989) we let the set of issues $A$ be represented by the unit sphere in $\mathbb{R}^{k}$, i.e.,

$$
A=\left\{a \in \mathbb{R}^{k}:\|a\|=1\right\},
$$

where $\|\cdot\|$ is the Euclidean distance, and we interpret the inner product $p^{i} \cdot a$ as a measure of the attractiveness of issue $a \in A$ for a player with position $p^{i}$. More precisely, we interpret the inequality $p^{i} \cdot a \leq p^{j} \cdot a$ as player $i$ being more in favor of issue $a \in A$ than player $j$. For a spatial game $g=(v, p)$ and an issue $a \in A$ we let $S_{g}(a) \in P(N)$ be a minimal coalition such that $v\left(S_{g}(a)\right)=1$ and $p^{i} \cdot a \leq p^{j} \cdot a$ for all $i \in S$ and $j \in N \backslash S$. We assume that $A$ is endowed with the relative topology induced by the Euclidean topology on $\mathbb{R}^{k}$. Clearly, $S_{g}(a)$ is unique for almost all $a \in A$. Thus, $S_{g}(a)$ is the minimal winning coalition such that all players in $S_{g}(a)$ are more in favor of $a$ than all players not in $S_{g}(a)$. We call $S=S_{g}(a)$ minimal winning for a. For every coalition $S \in P_{0}(N)$ define $A_{g}(S)=\{a \in A: S(a)=S\}$, and define $\lambda_{g}(S)=\lambda\left(A_{g}(S)\right) / \lambda(A)$, where $\lambda(B)$ is the Lebesgue measure of a subset $B$ of $A$. In other words, $\lambda_{g}(S)$ is the fraction of issues for which the coalition $S$ is minimal winning. ${ }^{6}$

\footnotetext{
${ }^{6}$ We assume that measures on $A$ are defined with respect to Borel sets; note that all our results above in Section 5 are valid for the $\sigma$-field of Borel sets instead of $P(A)$.
} 
We now define the spatial power index $\Omega$ by

$$
\Omega(g)=S h\left(\sum_{S \in P_{0}(N)} \lambda_{g}(S) u_{S}\right)
$$

for every spatial game $g$, where the simple game $u_{S}$ is the unanimity game for $S$, i.e., $u_{S}(T)=1$ if and only if $S \subseteq T$ for all $T \in P(N)$. Clearly, $\Omega_{i}(g) \geq 0$ and $\sum_{j \in N} \Omega_{j}(g)=1$ for all $g$ and $i \in N$.

Next, for a spatial game $g=(v, p)$ we define $E_{g}: P(N) \rightarrow P(\mathcal{T})$ as follows: $E_{g}(\emptyset)=\emptyset$ and for every $S \in P_{0}(N)$ and $B \in \mathcal{T}, B \in E_{g}(S)$ if there is $T \subseteq S$ and $a \in B$ such that $T=S_{g}(a)$. Then $E_{g}$ is monotonic by definition and superadditive since $v$ is proper. Let the probability measure $\mu$ on the Borel sets $B$ of $A$ be defined by $\mu(B)=\lambda(B) / \lambda(A)$, i.e., $\mu$ is the uniform measure on $A$. We then have:

Theorem 5.6. For every spatial game $g=(v, p)$,

$$
\Omega(g)=\Phi^{\mu}\left(E_{g}\right)=\int_{A} S h_{i}\left(v_{\{a\}}^{E_{g}}\right) d \mu(a) .
$$

Remark 5.7. The Owen-Shapley spatial power index $O$ is based on a similar construction as above. However, for each issue $a \in A$ we say that the player $i \in S_{g}(a)$ with $p^{j} \cdot a \leq p^{i} \cdot a$ for all $j \in S(a)$ is pivotal for $a \in A$. Then $O$ assigns to player $i$ the fraction of all issues for which this player is pivotal. In other words, all 'power' of the coalition $S(a)$ accrues to the 'last' player to enter the coalition. We can then, similarly, define an effectivity function based on player $i$ being effective for $\{a\}$ whenever $i$ is pivotal for $a$. Such an effectivity function, however, is no longer superadditive. On the other hand, all our main results and in particular Theorem 5.4 also hold without requiring superadditivity: in fact, proofs in general become simpler since then the union of effectivity functions is always an effectivity function. Thus, we obtain a result similar to Theorem 5.6 for the Owen-Shapley spatial power index, and Theorem 5.4 still applies.

\section{A Lemma A.1}

The following lemma is used in the proof of Lemma 4.3.

Lemma A.1. Let $B \in P(A)$, let $I \subseteq \mathbb{N}$ with $|I| \leq 2^{|A|}$, and for each $j \in I$ let $S_{j} \in P(N)$. Let $E=\bigcup_{j \in I} U_{S_{j}}^{B}$. Then $E \in \mathcal{E}$ and

$$
\varphi(E)=\sum_{\emptyset \neq J \subseteq I}(-1)^{|J|+1} \varphi\left(\bigcap_{j \in J} U_{S_{j}}^{B}\right) .
$$

Proof. It is straightforward to check that $E \in \mathcal{E}$. We prove the second statement by induction on $|I|$. The statement obviously holds for $|I|=1$. Let $\ell \geq 2$ 
and suppose the statement is true when $|I|<\ell$. Let $I=\{1, \ldots, \ell\}$. Then

$$
\begin{aligned}
\varphi(E)= & \varphi\left(U_{S_{1}}^{B}\right)+\varphi\left(\bigcup_{j=2}^{\ell} U_{S_{j}}^{B}\right)-\varphi\left(U_{S_{1}}^{B} \cap\left(\bigcup_{j=2}^{\ell} U_{S_{j}}^{B}\right)\right) \\
= & \varphi\left(U_{S_{1}}^{B}\right)+\sum_{\emptyset \neq J \subseteq\{2, \ldots, \ell\}}(-1)^{|J|+1} \varphi\left(\bigcap_{j \in J} U_{S_{j}}^{B}\right) \\
& \quad-\sum_{\emptyset \neq J \subseteq\{2, \ldots, \ell\}}(-1)^{|J|+1} \varphi\left(U_{S_{1}}^{B} \cap\left(\bigcap_{j \in J} U_{S_{j}}^{B}\right)\right) \\
= & \sum_{\emptyset \neq J \subseteq I}(-1)^{|J|+1} \varphi\left(\bigcap_{j \in J} U_{S_{j}}^{B}\right),
\end{aligned}
$$

where the first equality follows from the transfer property and Lemma 2.2; the second equality follows by induction; and the third equality follows by straightforward rewriting.

\section{B Independence of the axioms}

The following lemma shows the independence of the axioms in Theorems 4.5, 4.9 , and 4.10 .

Lemma B.1. There are power indices $\varphi^{1}, \varphi^{2}, \varphi^{3}, \varphi^{4}$ such that

1. $\varphi^{1}$ satisfies the Transfer Property, Anonymity, Strong Monotonicity, but not Neutrality;

2. $\varphi^{2}$ satisfies the Transfer Property, Anonymity, Neutrality, but not Monotonicity;

3. $\varphi^{3}$ satisfies the Transfer Property, Strong Monotonicity, Neutrality, but not Anonymity;

4. $\varphi^{4}$ satisfies Anonymity, Strong Monotonicity, Neutrality, but not the Transfer Property.

\section{Proof.}

1. Let $\varphi^{1}=\Phi^{\omega}$ where $\omega$ is a non-negative weight system satisfying $\omega^{B}=0$ for all $B \subseteq A$ with $|B| \geq 2$ and $\omega^{\{a\}} \neq \omega^{\{b\}}$ for some $a \neq b$. Then $\varphi^{1}$ has the desired properties by Theorem 4.10 , and $\varphi^{1}$ is not neutral by Theorem 4.9 .

2. Let $\varphi^{2}=\Phi^{\omega}$ where the weight system $\omega$ satisfies $\omega^{B}=\omega^{B^{\prime}}$ if $|B|=\left|B^{\prime}\right|$, $\omega^{B} \leq \omega^{B^{\prime}}$ if $B^{\prime} \subseteq B$, and $\omega^{B}<0$ for some $B \subseteq A$. Then $\varphi^{2}$ has the desired properties but is not monotonic by (the proof of) Theorem 4.5 and by Theorem 4.9 . 
3. Let $\alpha=\left(\alpha_{i}\right)_{i \in N}$ be a vector of strictly positive and distinct real numbers with $\sum_{i \in N} \alpha_{i}=1$, and let $S h^{\alpha}$ be the weighted Shapley value (Shapley, 1953a; Kalai and Samet, 1987). Then $\varphi^{3}(E)=\sum_{a \in A} \frac{1}{|A|} S h^{\alpha}\left(v_{\{a\}}^{E}\right)$ satisfies the Transfer Property, Strong Monotonicity, and Neutrality because of the respective properties of the weighted Shapley value, and it is clear that $\varphi$ is not anonymous.

4. Let $N=\{1,2,3\}$, let $B z$ denote the normalized Banzhaf-Coleman index (e.g. Owen, 1995), and let $\varphi^{4}(E)=\sum_{a \in A} \omega^{a} B z\left(v_{\{a\}}^{E}\right)$, where $\omega$ is a nonnegative weight system such that $\omega^{B}=0$ for all $B \subseteq A$ with $|B| \geq 2$ and $\omega^{\{a\}}=\frac{1}{|A|}$. Then $\varphi^{4}$ violates the Transfer Property but satisfies all the other properties.

The independence of the Axioms in Theorems 4.5 and 4.10 follows from points $2-4$; and the independence of the axioms in Theorem 4.9 follows from points 1-4 in Lemma B.1.

The next lemma shows that the axioms in Theorem 5.4 are independent.

Lemma B.2. There are power indices $\varphi^{1}, \ldots, \varphi^{5}$ such that

1. $\varphi^{1}$ satisfies Anonymity, Strong Monotonicity, Continuity, Null Player, but not the Transfer Property;

2. $\varphi^{2}$ satisfies the Transfer Property, Strong Monotonicity, Continuity, Null Player, but not Anonymity;

3. $\varphi^{3}$ satisfies the Transfer Property, Anonymity, Continuity, Null Player, but not Strong Monotonicity;

4. $\varphi^{4}$ satisfies the Transfer Property, Anonymity, Strong Monotonicity, Null Player, but not Continuity;

5. $\varphi^{5}$ satisfies the Transfer Property, Anonymity, Strong Monotonicity, Continuity, but not Null Player.

\section{Proof.}

1. Let $N=\{1,2,3\}$ and let $\varphi^{1}(E)=\int_{A} B z\left(v_{\{a\}}^{E}\right) d \mu(a)$ for every $E \in \mathcal{E}$, where $B z$ is the Banzhaf-Coleman index as in Lemma B.1, and $\mu$ is a probability measure on $P(A)$.

2. Let $\varphi^{2}(E)=\int_{A} S h^{\alpha}\left(v_{\{a\}}^{E}\right) d \mu(a)$ for every $E \in \mathcal{E}$, where $S h^{\alpha}$ is a weighted Shapley value with all weights distinct as in Lemma B.1, and $\mu$ is a probability measure on $P(A)$.

3. Let $\mu$ be a (signed) measure on $A$ with $\mu(A)=1$ and $\mu(B)<0$ for some $B \in P(A)$. Then let $\varphi^{3}(E)=\int_{A} S h\left(v_{\{a\}}^{E}\right) d \mu(a)$ for every $E \in \mathcal{E}$. 
4. Let $\varphi^{4}(E)=\int_{A} \operatorname{Sh}\left(v_{\{a\}}^{E}\right) d \mu(a)$ for every $E \in \mathcal{E}$, where $A=[0, \infty)$ and $\mu(B)=\lim _{k \rightarrow \infty} \frac{1}{k} \cdot \lambda(B \cap(0, k))$ for every Borel set $B$ of $A$, where $\lambda$ is the Lebesgue measure. Observe that $\mu$ is finitely additive but not $\sigma$ additive, but the integral is still well-defined since the integrand takes only finitely many values. Now $\varphi^{4}$ satisfies all axioms except CO by the proof of Theorem 5.4.

5. Let $\varphi_{i}^{5}(E)=\frac{1}{n}$ for every $i \in N$.

\section{References}

Bertini, C., Freixas, J., Gambarelli, G., Stach, I., 2013. Comparing power indices. International Game Theory Review 15.

Crama, Y., Leruth, L., 2007. Control and voting power in corporate networks: Concepts and computational aspects. European Journal of Operations Research $178,879-893$.

Crama, Y., Leruth, L., 2013. Power indices and the measurement of control in corporate structures. International Game Theory Review 15.

Dubey, P., 1975. On the Uniqueness of the Shapley Value. International Journal of Game Theory 4, 131-139.

Enelow, J.M., Hinich, M.J., 1984. The Spatial Theory of Voting: An Introduction. Cambridge University Press.

Enelow, J.M., Hinich, M.J. (Eds.), 1990. Advances in the Spatial Theory of Voting. Cambridge University Press.

Grofman, B., Owen, G., Noviello, N., Glazer, A., 1987. Stability and centrality of legislative choice in the spatial context. The American Political Science Review 81, 539-553.

Kalai, E., Samet, D., 1987. On weightedshapleyvalues. The International Journal of Game Theory 16, 205-222.

Karos, D., Peters, H., 2015. Indirect control and power in mutual control structures. Games and Economic Behavior 92, 150-165.

Martin, M., Nganmeni, Z., Tchantcho, B., 2014. The owen and shapley spatial power indices: a comparison and a generalization. Working paper, THEMA, Cergy Pontoise.

Moulin, H., Peleg, B., 1982. Cores of effectivity functions and implementation theory. The Journal of Mathematical Economics 10, 115-145.

Owen, G., 1995. Game Theory (3rd edition). Academic Press. 
Owen, G., Shapley, L.S., 1989. Optimal location of candidates in ideological space. International Journal of Game Theory 18, 339-356.

Peters, H., Zarzuelo, J.M., 2016. An axiomatic characterization of the owenshapley spatial power index. The International Journal of Game Theory 14, $1-21$.

Shapley, L.S., 1953a. Additive and non-additive set functions. PhD Thesis Princeton University.

Shapley, L.S., 1953b. A value for n-person games, in: Kuhn, H., Tucker, A. (Eds.), Contributions to the Theory of Games. Princeton University Press, pp. 307-317.

Shapley, L.S., Shubik, M., 1954. A Method for Evaluating the Distribution of Power in a Committee System. The American Political Science Review 48, 787-792. 